\title{
Tilt Rotor Aircraft Coupling Research
}

\author{
Chong Zhao ${ }^{1, a}$ and Ning Wang ${ }^{1, b}$ \\ ${ }^{1}$ Xijing University, Shaanxi Xi'an, 710123, China \\ a2571449451@qq.com, b3467798622@qq.com
}

Keywords: Nonlinear; Linear Mode; Simulation; Coupling

\begin{abstract}
Through the rotor machine balancing linearization, the nonlinear model of this paper on the basis of the linear model, through the simulation analysis model of dynamic coupling and coupling operation, through the characteristic root configuration method for inner loop decoupling control, realize the dynamic decoupling.
\end{abstract}

\section{Introduction}

Open-loop characteristics from a helicopter, the helicopter has strong coupling characteristics. And tilt rotor aircraft, including helicopters mode, therefore also has strong coupling. Coupling of tilt rotor aircraft has two forms, one caused by the system matrix, another caused by control matrix, the paper said caused by coupling of system matrix for the dynamic coupling, coupling caused by control matrix for the manipulation of the coupling.

In mathematical sense, manipulation of the coupling is zero initial response caused by the external input of a process. From a physical sense, the manipulation of the coupling is mainly caused by the tilt rotor aircraft special pneumatic layout. Such as longitudinal cycle variable pitch change, inevitably causes the rotor aerodynamic torque in the size of the roll channel and channel. Equivalent in roll channel and the total distance on the manipulated variable. For tilt rotor aircraft, due to the effect of joint of the system matrix and matrix. Control coupling inspire dynamic coupling, therefore, the tilt rotor aircraft open-loop control response of the coupling degree is very serious, according to the characteristics of the two coupling control system design will use different strategies, the dynamic coupling can be effectively solved, by state feedback control coupling can be solved by pre compensation.

\section{Tilt Rotor Aircraft Nonzero Input Dynamic Response Analysis}

In order to more intuitive reflect FGC characteristics of tilt rotor aircraft can respectively under different nacelle Angle analysis of the zero input response and zero state response.

The so-called zero input response is given under the condition of manipulated variable is zero, inspection system for the dynamic response of the nonzero initial value. Four kinds of circumstances.

(1) The vertical velocity is equal to the rest of the state variable is zero dynamic response.

(2) The roll Angle is equal to the speed, the remaining quantity of state is zero dynamic response.

(3) The pitching Angle is equal to the speed, the remaining quantity of state is zero dynamic response.

(4) The yaw angular velocity equal to zero., the rest of the state variable is zero dynamic response.

Analysis of tilt rotor aircraft, helicopter mode zero input response, its response diagram is as follows: 


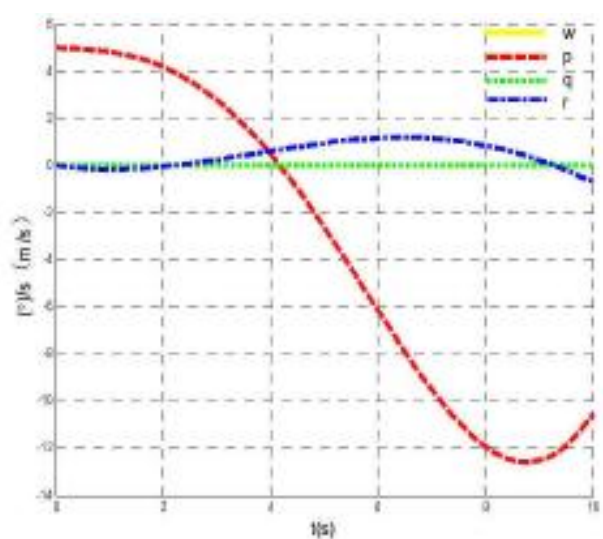

Figure 1. Vertical ring dynamic response speed nonzero initial value

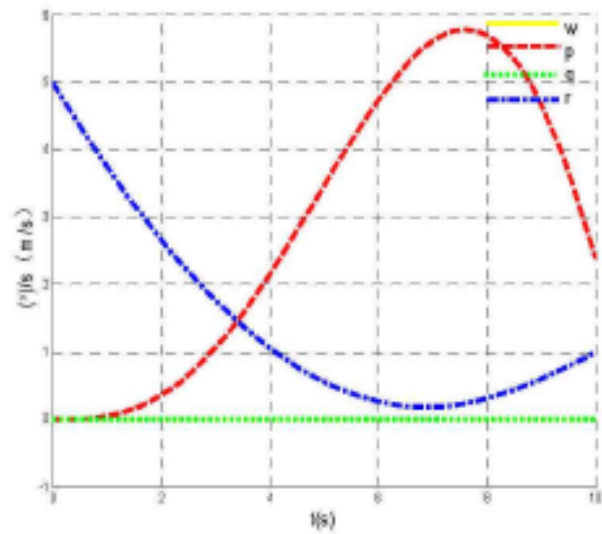

Figure 3. Pitching Angle speed q nonzero initial value dynamic response

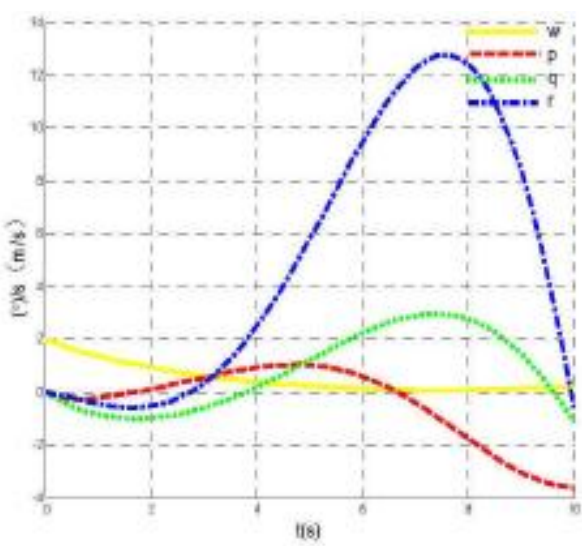

Figure 2. Roll speed dynamic responses of the nonzero initial value

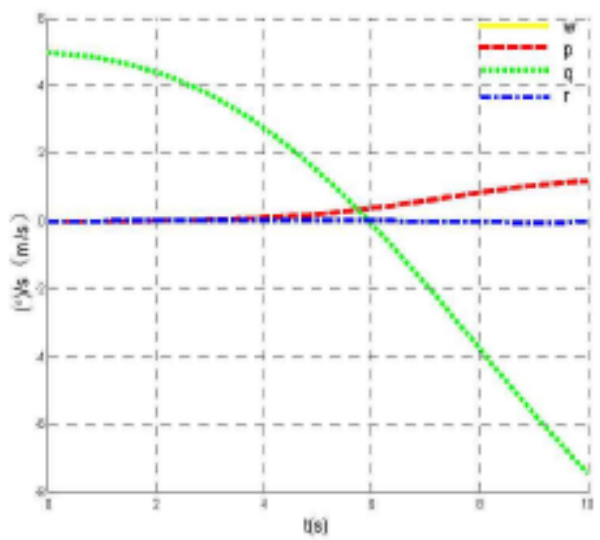

Figure 4. Bucket yaw angular velocity dynamic ring r nonzero initial value

From Fig. 1 to Fig. 4 helicopter mode zero input dynamic response can be seen that the vertical speed, pitch Angle and roll Angle speed coupling to the yaw angular velocity are very significant, vertical coupling between speed and the roll Angle is serious, and the mutual influence between speed of roll Angle and pitching Angle speed is not very serious. Horizontal channel and dynamic coupling between the longitudinal channels is relatively small.

Can be seen from the above zero input response figure, from the dynamic coupling, tilt rotor aircraft in the helicopter mode, the vertical speed, pitch Angle and roll Angle speed coupling to the yaw angular velocity are very significant, and the mutual influence between speed of roll Angle and pitching Angle speed is not very serious, this shows that the channel dynamic coupling between horizontal and vertical channel between the relatively small.

\section{Tilt Rotor Aircraft Nonzero State Dynamic Response Analysis}

Zero state response of state is the entire initial value is zero, the dynamic response of the inspection system to control the input, we can find that tilt rotor aircraft control coupling.

A helicopter hovering mode control response analysis, the helicopter model with helicopter control is given priority to, simply analyze the helicopter manipulated variable. 


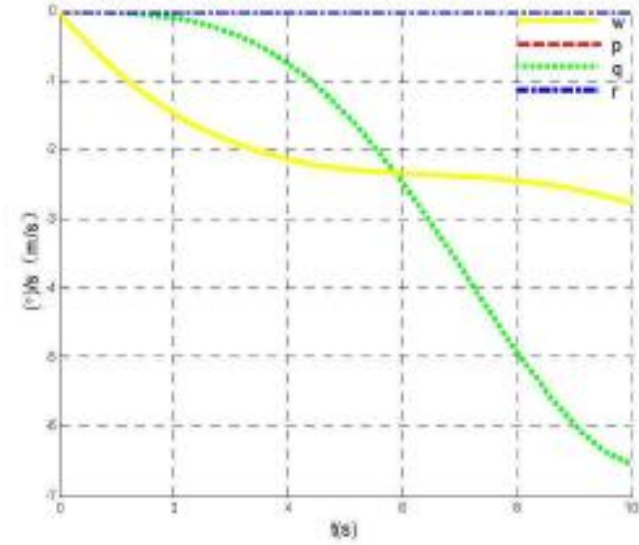

Figure 5. helicopters hovering mode from the step response

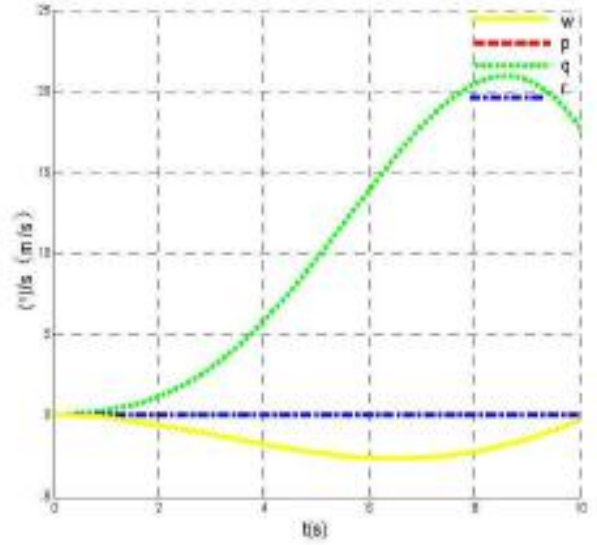

Figure 6. helicopter hovering mode vertical cycle variable pitch step response

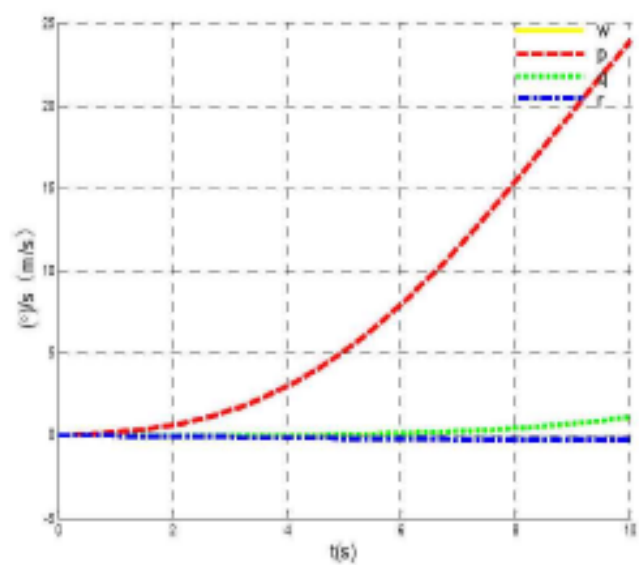

Figure 7. hovering mode transverse periodic

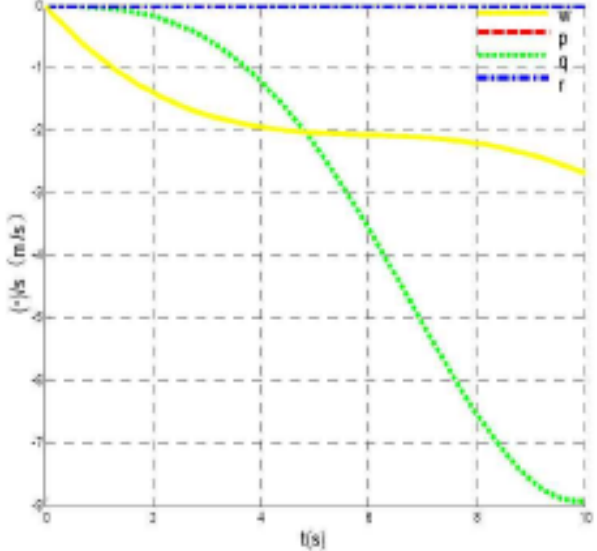

Figure 8. level flight from the unit step response variable pitch unit step response

Can be seen from the Fig. 5 to Fig. 8, the total is apart from the manipulation of the main influence on the speed of pitching Angle, because the total distance manipulated variable change, will make the tilt rotor aircraft up torque change, which affects the pitching Angle speed changes. Total distance control and engine throttle control thrust together, thus the vertical speed will change too. Longitudinal cycle variable pitch before and after make the rotor wheel dump, so as to change the direction of the force of rotor, and thus change the pitching Angle velocity of the plane. But at the same time, it will cause the change of the vertical velocity. Transverse cycle variable pitch is mainly used for aircraft roll control.

In the manipulation of the coupling, the total distance control mainly change the vertical speed, at the same time, the yaw angular rate of the strong coupling lateral cycle variable pitch control main change roll Angle rate, at the same time cause the pitching Angle rate highly coupled longitudinal cycle variable pitch control main effect on rate of pitching Angle, at the same time, the height of the roll Angle rate caused by coupling the rudder mainly cause the changes of yaw Angle, at the same time for roll angular velocity has the strong coupling, coupling of vertical velocity also has a certain effect. To manipulate the coupling, the coupling degree is still has the characteristics of the changes over the speed. Overall, tilting rotor machine roll channel and pitch channel of mutual coupling is serious, and the roll channel and pitch channel of course channels and highly that almost no coupling height channels of course there are larger coupling, and the roll channel and pitch channel coupling is smaller.

Tilt rotor aircraft in the helicopter mode, the manipulation of the channel control response is not stable, operate the flight simulator shows that separate one when the channel response is not stable, the thrust control channel control response is unstable with the increase of the speed. Along with the 
To the fixed wing aircraft model transformation, main control response of the control channel gradually stable. In the design of tilt rotor aircraft control system when it is worth noting that as the tilt rotor aircraft flight mode conversion, thrust in control of the main control channel response by axial velocity transformation for the axial velocity.

\section{Summary}

For rotor aircraft flying nonlinear model for multiple steady state of balancing calculation, preliminary understanding the control rule of tilt rotor aircraft. In balancing the results on the basis of the control response calculations, that tilt rotor aircraft in the helicopter mode, to manipulate single channel response is unstable in airplane mode, while the control response is stable, but on the pitch channel and the yaw channel the oscillation behavior of the control response is very serious. Therefore, must have a good control performance.

\section{References}

[1] P.D. Talbot, B.E.Tinling,WA. Decker etal. A Mathematical Model of a single Main RotorHelicopter for piloted simulation. NASA-TM-84281, 1982.

[2] R.L.Marr, K.WSambell, GT.Nea1. V/stol tilt rotor study-volume VI Hover, Low speed and conversion tests of a tilt rotor aeroelastic model. NASA CR 114615. 1973.

[3] Bernard Mettler. Modeling small-scale unmanned rotorcraft for advanced flight control design,Ph.D. Dissertation. Pennsylvania, Carnegie mellon university, 2001.

[4] Anna Kathleen Tyler Howard. The Aero-mechanical stability of softinplane tiltrotors Ph.D.Dissertation, Aerospace Engineering, 2001.

[5] Dr.Anthony, J.Calise and Rolf T.Rysdyk. Adaptive model inversion filght control for tiltrotor aircraft. AIAA, 1997, AIAA-97-3758:1633-1639 